\title{
Dementia Patient Activity Monitoring and Fall Detection using IOT for Elderly
}

\author{
Prof. Deepali K. Shende, Mr. Sidheshwar Madrewar'2, Mr. Shivam Bhongade², Mr. Shivam Dugade ${ }^{2}$ \\ ${ }^{1}$ Assistant Professor, ${ }^{2}$ Students \\ Electronics and Telecommunication department, Savitribai Phule Pune University, \\ Sinhgad Institute of Technology, Kusgaon (BK), Lonavala, Pune, India
}

\begin{abstract}
How to cite this paper: Prof. Deepali K. Shende | Mr. Sidheshwar Madrewar | Mr. Shivam Bhongade | Mr. Shivam Dugade "Dementia Patient Activity Monitoring and Fall Detection using IOT for Elderly" Published in International Journal of Trend in Scientific Research and Development (ijtsrd), ISSN: 24566470, Volume-3 | Issue-4, June 2019, pp.139-143, URL: https://www.ijtsrd. com/papers/ijtsrd2 3606.pdf

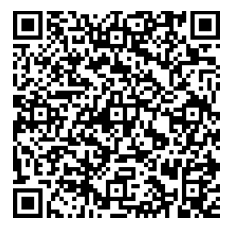

IITSRD23656

Copyright (C) 2019 by author(s) and International Journal of Trend in Scientific Research and Development Journal. This is an Open Access article distributed under

the terms of the

Creative

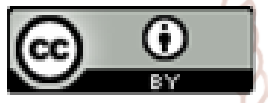

Commons Attribution License (CC BY 4.0)(http://creativecommons.org/licens es/by/4.0)
\end{abstract}

\section{ABSTRACT}

There is drastic increase in population of dementia patients and also elderly since we are growing as modern society. To avoid the need of special care centers for people suffering from such disease usually it is suggested to stay in their own house. The product presented in this paper provides user current localization, Real-time image processing, automatic fall detection and activity monitoring and also manage emergency situations. The Product aims to help patients retain their independence, whilst reducing the demand on care givers as well as providing patients freedom of independently walks outside.

Keywords: Dementia, User localization, Real time Image, Automatic fall detection, Activity monitoring.

\section{INTRODUCTION}

A Dementia is the loss of cognitive functioning. This effects the person's daily life and activities such as the ability to think, remember and reason. Alzheimer's disease, the most common form of dementia, is progressive and slowly destroys the brain limiting functionality, decreasing quality of life and eventually leading to death. Assistive technologies are continuously being developed and utilized to help those suffering from dementia perform tasks, which they would not normally be able to perform. These technologies can make vast differences to the quality of their lives, such as prolonging the length a Dementia patient can live in their own home and also help them to complete their tasks independently which otherwise they would have forgot to do.
This project proposes a safety assistant, named wandering path tracking and fall detection system (PTFaD), which are a smartphone-based system monitoring people with dementia for both indoor and outdoor. The whole system comprises wandering path tracking and fall detection, safety-zone monitoring, communication services, alert notifications, and emergency medical services. To effectively track the elderly, the PTFaD uses a smartphone camera to take real-time pictures along the user's path as he or she moves about. Those photos, along with time and GPS signals, are delivered to and stored on the Cloud system. When there is a need, family caregivers can download those data to quickly find a way to help the elderly individual. Additionally, this study uses tri-axial accelerometers to examine falls.

When a fall incident is detected, the alarm system issues notification messages to all emergency contact person in sequence until one responds to the system. If no one

responds within a pre-determined time period, then the system immediately calls emergency medical services to procure timely help for that elderly individual. To assure individuals' data is safeguarded appropriately, an RSA method has been adopted by the system to encrypt stored data. The purpose is to guarantee that personal privacy is well protected. Any attempt at unauthorized access to the smartphone or Cloud will not succeed. Our reliable and minimally intrusive system provides people with dementia with an opportunity to improve their quality of lives and also maintain their social networks.

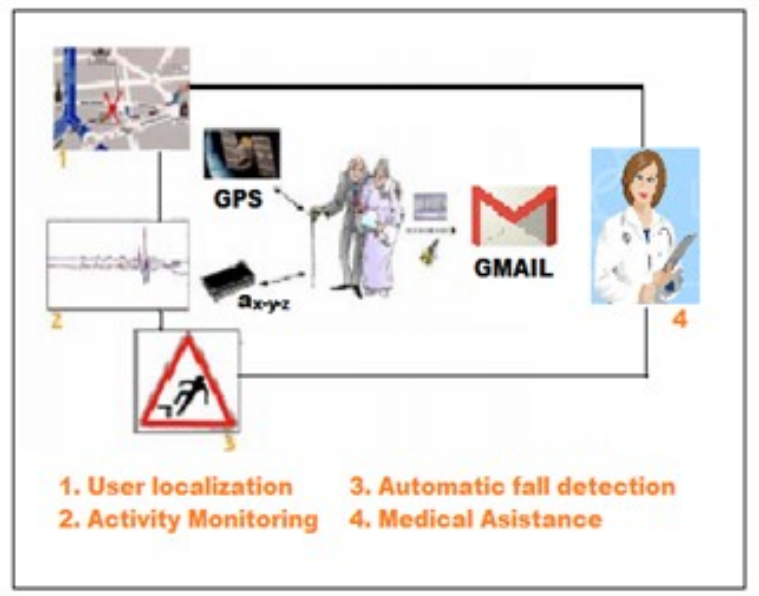

Fig. 1- Services offered by the system 


\section{DEMENTIA AND ITS SYMPTOMS}

A Dementia is not a single illness. It is a group of symptoms caused by specific brain disorders. The most common cause is Alzheimer's disease, but dementia can also be the result of a stroke or mini-strokes. The main symptoms are:

1. Loss of memory - such as forgetting the way home from the shops, forgetting names or places, or being unable to remember what happened earlier in the day.

2. Mood changes - because of damage to parts of the brain that control emotions, people can become frightened, angry or sad more easily.

3. Communication problems - a decline in the ability to talk with someone also to read and write something.

Dementia is progressive - the symptoms will get worse and worse with time. Although there is no cure for this, treatments can slow the progression of the disease and also there are different ways of helping to keep it manageable. Definitely we need a care taker to keep watch on these dementia patients, and to make healthier life of dementia patient we all focusing on technology to help us.

\section{FALL RISK FACTORS}

A person can be more or less prone to fall, depending on a number of risk factors and hence a classification based on only age as a parameter is not enough. In fact, medical studies have determined a set of so called risk factors:

\section{$>$ Intrinsic:}

Age (over 65)

Low mobility and bone fragility

Poor balance

Chronic disease

Cognitive and dementia problems

Parkinson disease

Sight problems

Use of drugs that affect the mind

Incorrect lifestyle (inactivity, use of alcohol, obesity)

Previous falls

$>$ Extrinsic:

Individual (incorrect use of shoes and clothes)

Drugs cocktail

$>$ Internal Environment:

Slipping floors

Stairs

Need to reach high objects

$>$ External Environment:

Damaged roads

Crowded places

Dangerous steps

Poor lighting

There is a clear correlation between the above list and the probability of fall \& the number of people that fall is as follows:

$>\quad 8 \%$ of people without any of risk factors

$>28 \%$ of people with only one risk factor

$>77 \%$ of people with four or more risk factors

The history of the falls is also important since people who have already fallen few times are more at risk falling again. This can be due to psychological (fear, shame, loss of selfesteem), and/or physical (injuries, lack of exercise) reasons.

\section{HOW, WHERE \& WHY PEOPLE FALL}

Among all half of the falls take place near or inside the house. Usually women fall in the kitchen whereas men fall in the garden.
The rate of falls increases significantly among elderly people living in nursing homes: at least $40 \%$ of the patients fell twice or more within 6 months. This rate is five times more with respect to the rate of fall when people live at home. This may be due to people having to adjust themselves with the new living environment and its obstacles.

\subsection{PHYSICAL CAUSES}

The factors that lead to most of the falls in people over the age of 60 are to stumble on obstacles or steps and to slip on a smooth surface. The fall is usually caused by loss of balance due to dizziness. Approximately $14 \%$ of people do not know why they fall and a smaller number of people state that the fall is due to the fragility of the lower limbs. Further group of researchers determined that traditional fall prevention measures such as bed rails can make the fall worse (Masud \& Morris, 2001).

\subsection{ACTIVITIES}

Mainly the activities of daily living (ADL) that involve a small loss of balance such as standing or walking are the reason to fall. Fewer falls happen during daily activities that involve a more significant movement such as sitting on a chair or climbing the stairs. Conversely, activities usually defined "dangerous", such as jogging or physical exercises are less likely to increase the probability of a fall (Tinetti et al., 1988).Number of falls during the day are more than during the night (Campbell et al., 1990).

\subsection{CONSEQUENCES}

Accidental falls are the main cause of admission in a hospital and the sixth cause of death for people over 65. For people aged between 65 and 75 accidental falls are the second cause of death and the first cause in those over 75 (Bradley et al., 2009).

\subsubsection{PHYSICAL DAMAGE}

Scratches and bruises are the soft injures which occur on fall (Bradley et al., 2009). In the worst cases the injuries are concentrated on the lower part of the body, mainly on the hip. On the upper part of the body the head and the trunk injuries are the most frequent. About $66 \%$ of admissions to an hospital are due to at least one fracture. The fracture of elbow and forearm are more frequent but hip fracture is the most difficult to recover from. Such a fracture in fact requires a long recovery period and involves the loss of mobility and independency. Sometimes, when a person falls and is not able to stand up by himself, he lies down on the floor for long time. This leads to additional health problems such as hypothermia, Depression, complications and in extreme cases can cause death (Lord et al., 2001).

\subsubsection{PSYCHOLOGICAL DAMAGE}

A fall also involves hidden damages that affect the selfconfidence of a person (Lord et al., 2001). Common consequences are low confidence, loss of independence, limited capabilities, loss of self esteem and generally, a lower quality of life.

\subsubsection{ECONOMICAL DAMAGE}

The direct costs associated with falls are due to the medical examinations, hospital recoveries, rehabilitation treatments, tools of aid (such as wheelchairs, canes etc.) and caregivers service cost (Englander \& Hodson, 1996).

Indirect costs concern the death of patients and their consequences. Recent studies have determined that in the 
year 2000 alone fall-related expenses was above 19 billion dollars and it is estimated to reach 54.9 billion in 2020 . This shows that year by year, health costs due to the falls are increasing dramatically (Massachusetts Department of Public Health, 2008).

\section{RELATED WORK}

The Today, there are an increasing number of technological devices on the market to help in the search and rescue of people with Alzheimer's disease or a related dementia who have become lost, whether from a home, long-term care residence or other location. There are pros and cons to each of these-active\| systems, and no service can guarantee that an individual can be found or found unharmed every timeunderscoring the need to deploy all possible methods of prevention to stop occurring in the first place. However, since the majority of people with Alzheimer's disease will wander away at some point during the disease, possibly despite the best precautions taken, systems should be in place to aid families and law enforcement in the event that a situation mandates a search and rescue.

Approximately 75 percent of people with Alzheimer's disease receive care at home. Noting the potential market, more companies have been developing technologicallyadvanced systems aimed at ensuring the safety of the person with dementia and the peace of mind of the family caregiver. Given the importance of ensuring the safety of residents in long-term care facilities as well, these systems - even if not marketed as such-can also be used for persons living in these types of settings.

\subsection{RELATED WORK IN TRACKING AND LOCATING DEMENTIA}

While most of the available products share some similar characteristics, there can be distinct differences among them regarding:

$>$ Fundamental location technology (i.e., GPS, cellular, radio; and potential signal transmission problems);

$>$ Degree and type of communication (i.e., instant or timespecific alerts; notifications to or access by one or multiple contacts; call, text or e-mail alerts);

$>$ Hardware (i.e., hand-held or wearable; size and weight; user-activated; battery life; risk of removal; waterproof/water-resistant);

$>$ Location tracking (i.e., constant, at regular intervals, when emergency-only; consumer/law enforcement involvement);

$>$ Involvement of third parties that assist in notification and search and rescue, such as call centers or law enforcement;

$>$ Cost (i.e., device, activation fee, monthly monitoring fee, other products like chargers, batteries);

$>$ Ongoing support (i.e., call center, affiliation with private company or nonprofit organization that provides training for public/law enforcement, and education or other resources to caregivers); and

$>$ Accuracy of location and success rates for search and safe return.

$>$ These entire technology products helping dementia patient to relieve stress, and helps them in living quality life.

\subsection{RELATED WORK IN FALL DETECTION}

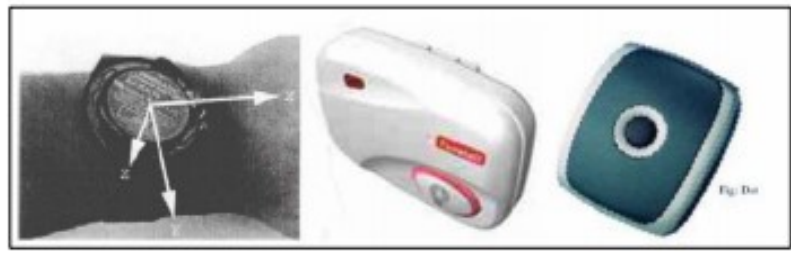

Fig.2 - Examples of fall detectors

As we dealing with fall detection of dementia patient, there is already lot of work done to find out human movement detection and fall detection. While previously different approaches suggested to monitor movement and detect fall the cameras were use to monitor on dementia patients. But as cost associate with this equipment is high, this approach is quite expensive. Second approach is to make use of accelerometer sensors. Sensors are attached with body so if there is sudden change each time patient moves or change in activity against to gravity it detects that movement and based on algorithmic approach it detects fall. Above discussed technology comes under category of wearable and non-wearable devices.

Fall detection systems currently use one of the below methods for sensing a fall condition:

$>$ Image recognition can be used to detect fall by placing cameras at overhead positions, thereafter learning movement patterns of person. The system becomes adaptive to the locations where human enters the room and remains stationary.(conditions of sitting on chair, lying on bed etc.). Common paths from point of entry to inactive areas are then remembered. If the person turns inactive in between the process of common path detection then fall gets detected and alerted.

Recognition by acoustic/vibration: This system detects fall condition by matching the obtained vibration from the floor against a set of known safe vibrations such as walking, running etc.

Worn sensor devices: This devices detects fall by analyzing data received from sensors which is worn by the user. Usually the sensors used are tri-axial accelerometer or gyroscope. If the data received from the sensors crosses a pre-defined threshold value then fall condition is detected.

$>$ Recognition by acceleration threshold: This system detects fall by analyzing acceleration reading receive from an accelerometer. If the amplitude of the acceleration crosses the lower and upper thresholds and if there is a change in position then fall condition is detected

With advancements in mobile technology smart phone prices have reduced significantly resulting in smart phones becoming easily affordable for all. Most of these smart phones have an in-built accelerometer which is generally used for user interaction and orientation detection. Same accelerometer can be re-used for fall detection, eliminating the need of any additional hardware or sensors and thereby reducing the cost involved. Smartphone also have the necessary capabilities for alerting in case of such emergency conditions of fall, by SMS, GPRS, call etc. GPS feature in smart phone can also be utilized to get the geographic location along with alert message making the alert more precise. 


\section{METHODS \& MATERIALS}

The mobile module will be worn at the belt, since this place has been defined like most discrete and convenient taking into account the size and weight of module. Its interface will include a "reset button" easily accessible to cancel an alarm if necessary or to set

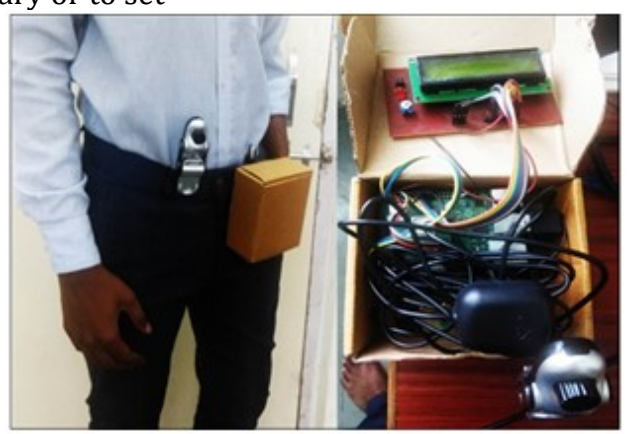

Fig.3. - System's Prototype

voluntarily an alarm. User location service is integrated on the system. The use of GPS technology allows total outdoor localization giving more flexibility in the use of the system. The function is necessary to complete main aspect that precise localization of the user in case of alarm is sent to send emergency services to the right spot.

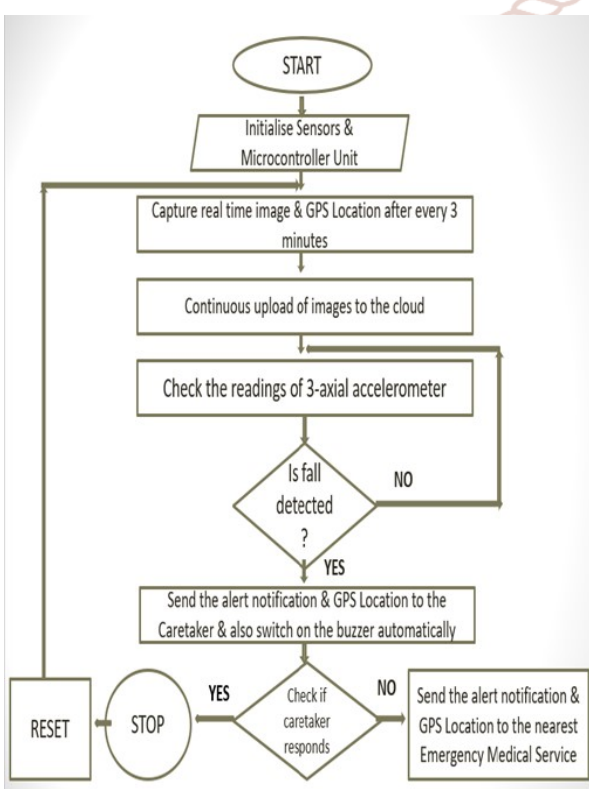

Fig.4.- System flow diagram

\subsection{FUNCTIONALITIES}

\subsubsection{AUTOMATIC FALL DETECTION}

This paper is basically concerned about monitoring and fall detection of dementia patients so they should not get lost. For fall detection we are using algorithm which is based on threshold values. This is most classical approach to fall detection. The accuracy of fall detection by this is high as compare to previously used algorithms.

Basically we have X, Y, Z axis orientation. And our algorithm is based on three axis acceleration; Acceleration in X-axis, Yaxis, Z-axis are represented by $\mathrm{Bx}$, By and Bz respectively. And equation is represented by,

$\mathrm{B}_{\text {sum }}=$ SquareRootof $\left(\mathrm{Bx}^{2}+\mathrm{By}^{2}+\mathrm{Bz}^{2}\right)$

We need to fix some threshold value and by comparing this threshold values we are analysing fall detection.

\subsubsection{USER LOCALIZATION}

In this system we are using GPS module to localize dementia patient by acquiring GPS co-ordinates and sending a caretaker a location in the form of link which direct a caretaker to Google maps to track down a dementia patient and to know the precise location we are also interfacing a camera module to capture real time image by which a caretaker can identify exact location of a dementia patient.

\section{RESULT \& CONCLUSION}

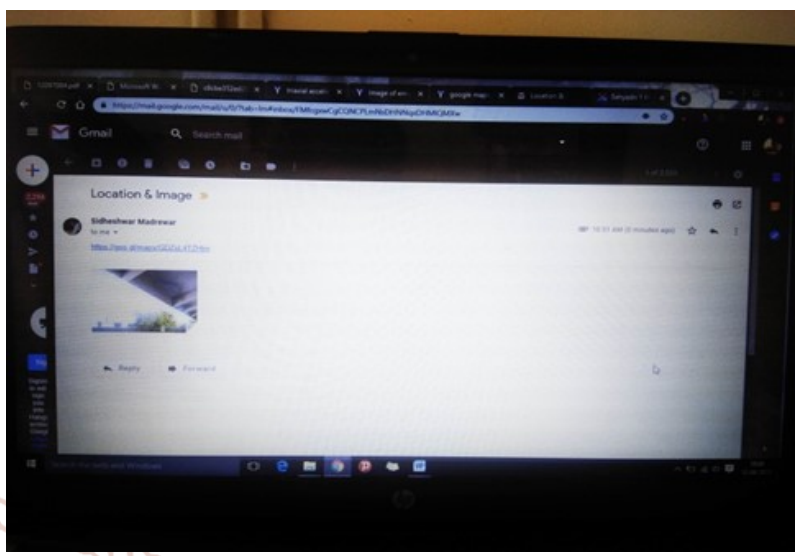

Fig. 5. - A Gmail received by care taker

Figure 5 shows sample of Gmail received by a care taker it displays a real time image $\&$ a link which direct to the Google map.

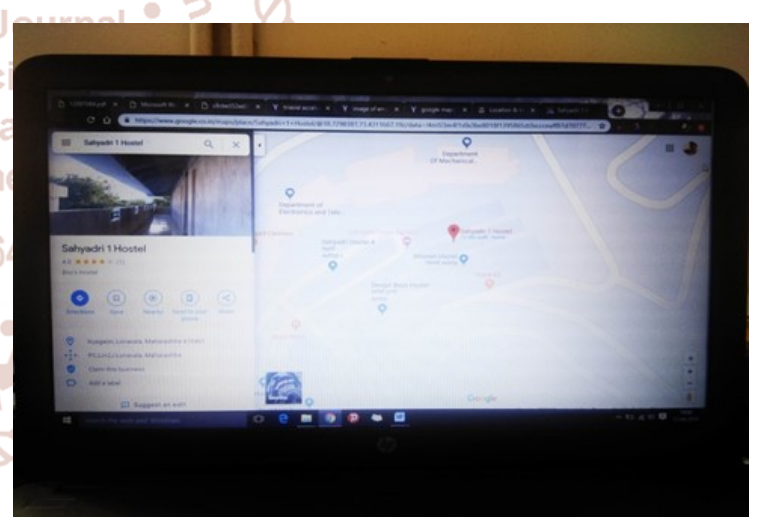

Fig. 6 - Location of dementia patient

Figure 6 shows a Google map which is showing a location of a dementia patient and it is acquired by opening a link which is provided in the Gmail by the system.

As the purpose of this paper to discuss the techniques available for monitoring patients and also discuss fall detection algorithm for them. This paper is focus mainly on implementation of system which will relieve stress on care taker and also enable dementia patient to perform there day to day life task without any interruption.

\section{REFERENCES}

[1] Shinde B. A., and Chawan P. M.(July 2014). Dementia patient movement detection and fall detection using smart phone technology. Retrieved from http://www.cs.odu.edu/ cs441/Papers/sense016.pdf 
[2] Abbate S., Avvenuti P., Vecchio A., Corsini P., and Light J.(n.d.). Monitoring of human movements for fall detection and activities recognition in elderly care using wireless sensor network: a survey. Retrieved from https://core.ac.uk/download/pdf/12097084.pdf

[3] Doughty, K.: Fall Prevention and Management Strategies Based on Intelligent Detection, Monitoring and Assessment. New Technologies in Medicine for the Elderly, Charing Cross Hospital (2000)

[4] Pérolle G., Fraisse P., Mavros M., and Etxeberria I.(n.d.). Automatic Fall Detection and Activity Monitoring for Elderly - Shaping the Future, IOS Press (2003) 798802
[5] Feld R., Bourke A., Nelson J. (n.d.). An Integrated Fall and Mobility Sensor andWireless Health Signs Monitoring System. Retrieved from https://www.researchgate.net/publication/22436001 0_An_integrated_fall_and_mobility_sensor_and_wireles s_health_signs_monitoring_system

[6] Noury, N., Virone, G., Barralon, P., Ye, J., Rialle, V., Demongeot, J.(n.d). New Trends in Health Smart Homes. IEEE2003 (2003)

[7] Fugger, E., Hochgatterer, A., Prazak, B.(n.d.). Integrated Alert \& Communication System for Independent Living of Older Adults. Assistive Technology - Shaping the Future, IOS Press (2003) 803-807. 